\title{
Survey of Conventional UUV Navigation Mapping Technique in Development of AQUA-X UUV
}

\author{
S. Faiz Ahmed, D. Hazry, M. Hassan Tanveer, M. Kamran Joyo, Faizan. A. Warsi, Khairunizam Wan, Zuradzman M. \\ Razlan, Shahriman A.B, Zunaidi IB, A.T. Hussain \\ Centre of Excellence For Unmanned Aerial Systems (COEUAS) Universiti Malaysia Perlis (UniMAP), \\ Seriab, 01000, Kangar, Perlis, Malaysia. \\ E-mail:syedfaiz@unimap.edu.my,hazry@unimap.edu.my,hassantanveer@live.com, \\ sky_kamran12@hotmail.com, _warsi128@hotmail.com, khairunizam@unimap.edu.my, abadal@unimap.edu.my \\ zunaidi@tatiuc.edu.my, shahriman@unimap.edu.my,abadal@unimap.edu.my
}

\begin{abstract}
Unmanned Underwater Vehicles (UUV) are devices that perform task with slight or no human interaction. These tasks are predefined set of commands called navigation methods, that are used to achieve desired mission goals. The navigation control system plays an important role in maneuvering of UUV and without it UUV cannot perform any mission task. In this research article a survey of different navigation techniques are presented for new design of Underwater mobile robot named as AQUA-X Robot, which uses four (4) propellers placed in cartesian coordinate configuration with parallel arrangement which help it in batter navigation maneuvering. The detail working principles, advantages and problems in different navigation techniques are thoroughly discussed. Besides that, their implementation on UUV on a ranged mission situation are also briefly described. Finally the accuracy of the different navigation techniques are compared and based on this comparison a most suitable navigation method is selected for developing AQUA-X UUV for better navigation.
\end{abstract}

Keywords: Underwater Vehicle, GPS, Acoustic, Navigation Technique

\section{Introduction}

Unmanned Underwater Vehicles (UUV) are the vehicles which can operate beneath the water with no or minor intervention of any human. Due to the advancements in sensor technologies the UUV's are rapidly becoming advanced and more reliable to be used in dangerous operations. These autonomous systems provide most likely option to fulfill the requirements of Navy operations. Armed forces use these vehicles for surveillance, intelligence and discovering underwater mines. One of the most challenging tasks is the development of navigation system for UUV. Navigation is the indication of position, attitude [16] and velocity of the vehicle relative to the reference coordinates at a given moment, it also encompasses plotting courses, avoiding obstacles and act and it is also defined as the measurement of the vehicle position within a prespecified grid [4]. This article is related to the navigational techniques used in UUV's which is then implemented in Aqua-X model.

Conventional UUV's use only propeller to produce thrust and actuated fins to navigate. The Aqua-X UUV consists of four fixed pitch propellers mounted at the four ends of an aerodynamic body frame as shown in fig.1. It uses a differential method to navigate under the water. 
D. Hazry, S. Faiz Ahmed, M. Hassan Tanveer, M. Kamran Joyo, Faizan. A. Warsi,

Khairunizam Wan, Zuradzman M. Razlan, Shahriman A.B, Zunaidi IB and A.T. Hussain

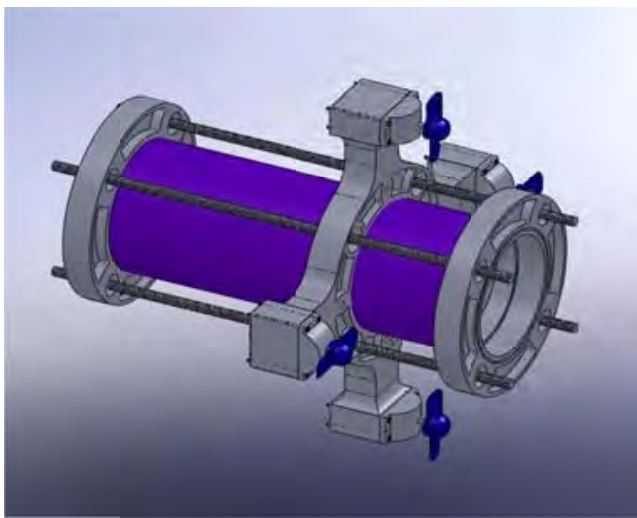

Fig.1. Aqua-X Model

\section{Navigation Techniques}

This section describes the navigation technique for the UUV and focus on how navigation method works. The operation of UUV is actually depending on its navigation to do a mission and operating. So the reliability of any UUV is depends up on the accuracy of the navigation system of that UUV. During maneuvering of UUV in an open sea environment, the accuracy of navigation is essential and should be good enough to bring it back to base station, otherwise the chance of losing the vehicle become very high. From the last two decades, many researcher works on development of different accurate navigation techniques for UUV [14], they used Vision method, Radar based, time base, acoustic, GPS and may more ways to developed more accurate navigation [15] method for different mission ranges. In this paper four different most famous techniques are presented.

\section{Dead Reckoning Navigation}

The most common approach in navigation technique is to implement the vehicle velocity in time to get the new estimate position of the UUV[9].

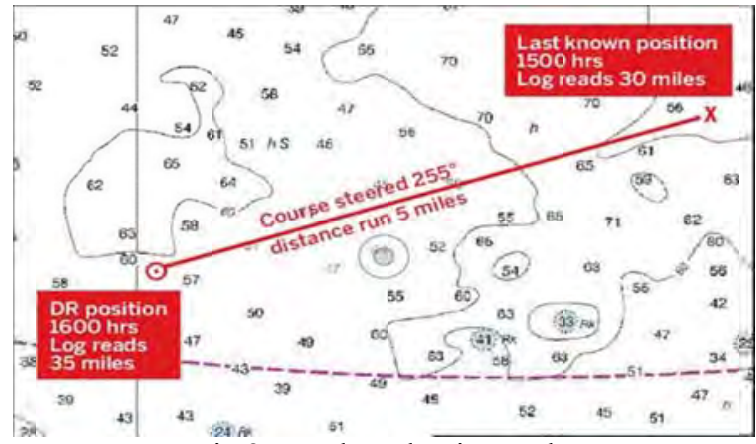

Fig.2. Dead Reckoning Path

Dead reckoning is the process of determining current position by projecting course and speed from a known past position, and predicting a future position by projecting course and speed from a known current position. The calculation of distance in this method is done by the compass and water speed sensor's feedback data. As this method is used, the external disturbance is added from the presence of an ocean current and that can drift the velocity component of the UUV and this deflection cannot be detected by water speed sensor. Fig. 2 shows the example of dead reckoning path calculation and it's slightly different from the actual path. For accurate distance measurement INS (Inertial Navigation System) is used, which provides very accurate directional information. The INS start with the double integration of acceleration sensed in the inertial space frame, and gives a relative position according to the start reference point. The quality of the data is depend on the precision of the INS sensor. The commercialize INS can update the position drift rates up to several kilometer per hour. Beside that the cost and power consumption made the INS system is not suitable for a small UUV.

During the operation near the bottom of the sea, Doppler Velocity Sonar (DVS) sensor is use to measure the relative between vehicle velocity to the ground. The DARPA UUB has achieved navigation performance of $0.01 \%$ of distance traveled using integrated of INS and DVS system [11]. Using this technique, the ultrasonic signals in downward oblique direction and determining by the change of echo frequency, usually for the good accuracy, four beam is use with angle near $30^{\circ}$ in four deferential direction. The trade-off in Doppler sonar's between accuracy, size and the maximum altitude, the higher frequency provide good accuracy and small transducer array, but the maximum latitude is slightly decreases. To increasing the accuracy the narrower beam width need to be use, but the size of the transducer is vice versa. The reference technique is use to get the 
velocity relative to the ground is the correlation sonar, the two dimensional sonar with a wide angle array is used to measure the reflection of the acoustic energy from the sea floor, the advantage of using this correlation sonar is focus on the large beam width.

\subsection{Dead Reckoning Advantage}

The advantage of this technique is that it requires no external reference in order to determine its position, orientation, or velocity once it has been initialized. It also can detect a change in its geographic position (a movement east or north for example), a change in its velocity (speed and direction of movement) and a change in its orientation (rotation about an axis). It's also measure the linear and angular acceleration applied to the system for good accuracy.

\subsection{Dead Reckoning Disadvantage}

All inertial navigation system are suffer from an integration drift, for example refer the Fig. 3 a small errors in the measurement of acceleration and angular velocity are integrated into a progressively larger errors in velocity, which are compounded into a still greater errors in position. Otherwise, the new position is calculated from previous calculated position and measured acceleration and angular velocity, this error accumulate roughly proportionally to the time since the initial position was input. So, the position must be periodically corrected by input from some other type of navigation.

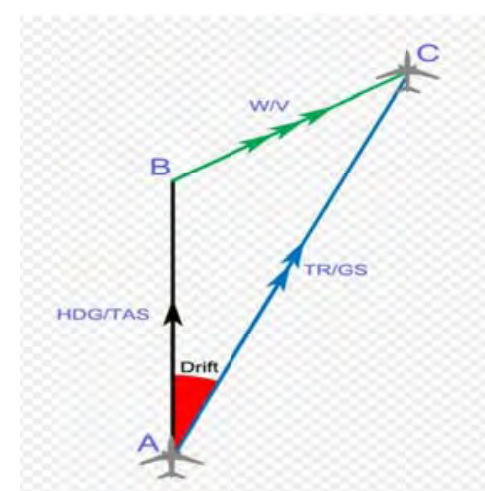

Fig.3. Drift that occur in Inertial Navigation System

\section{Acoustic Navigation}

The electromagnetic can be only propagate in the ocean at very low frequencies, but acoustic energy can be propagate well, so the research have made the acoustic transponder that can be use as beacons to give a guidance for UUV without need to be resurfacing. The acoustic navigation system consist of two sensor method which is: long baseline (LBL) and ultra short baseline (USBL) the application example shown in Fig. 4.

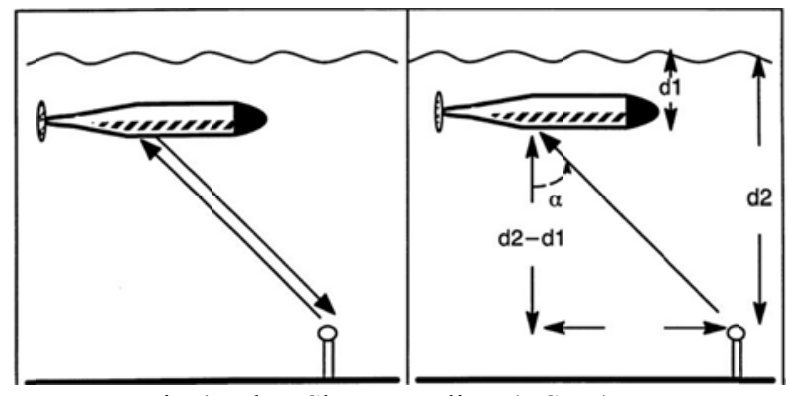

Fig.4. Ultra Short Baselines(USBL)

In Fig. 4 both of the system using an external transducer arrays to help navigation. In LBL navigation system, the array of transponders is deployed and survey into the position. Then the vehicle sends out an acoustic signal which is its later accept by bacon as receiver just like the ordinary formula of calculation using the ultrasonic, the actual location of UUV is determine by measuring the traveler time between the vehicle and beacon, this data can be manipulate relative distance between vehicle and each array node. This method facing a difficulty in acoustic environment, such as in shallow water or in the arctic, the rejection become difficult to filter between the direct arrival and multipath disturbance, and unacceptable of outliers .

This system is hyperbolic navigation, where the vehicle does not actively ping but instead listens to an array beacon where the geometry is known [12]. The beacon is ping in it sequence relative to other with it specific frequency. The vehicle can be relocated where it must be in space in order to hear the ping sequence as recorded. These techniques have greatly saved the power consumption of vehicle especially for multiple 
D. Hazry, S. Faiz Ahmed, M. Hassan Tanveer, M. Kamran Joyo, Faizan. A. Warsi,

Khairunizam Wan, Zuradzman M. Razlan, Shahriman A.B, Zunaidi IB and A.T. Hussain

UUV operation. Almost all LBL system work at the frequency of $10 \mathrm{kHz}$ and also give an accuracy in few meter with a maximum range on the order for a few kilometer, alternatively system operating at $300 \mathrm{KHz}$ have been created that can provide positioning repeatability down to 1 centimeter resolution in a triangular operating area that is 100 meter to a side.

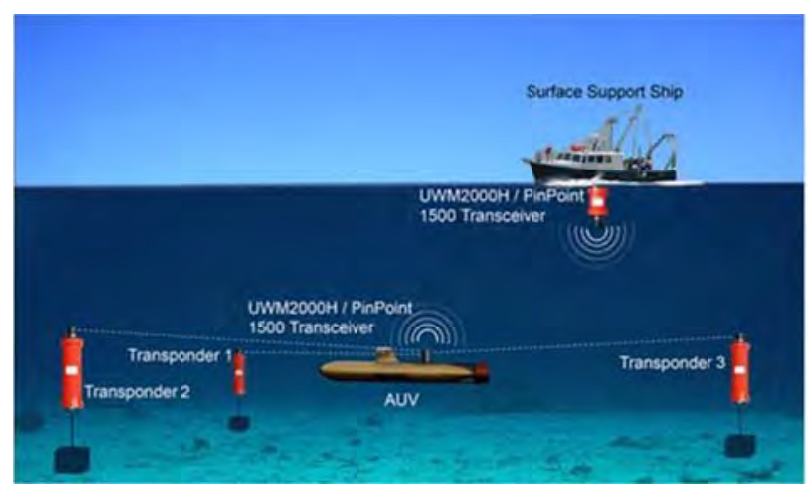

Fig. 5. Acoustic Navigation

Furthermore, USBL navigation in the UUV have a multi-element receiver array that allow it to measure the angle as well as the range to an acoustic beacon, this system is very popular and commercially use for tracking UUV from the ship. By measuring the interval time difference of a ping between hydrophone, the location of the vehicle to beacon is determined. It is essential to knowing the distance and direction to the beacon to allow for local navigation. Both LBL and USBL arrays com from various sources. The main sources of error come from two factors: error in assumed array geometry and errors in the assumed sound speed profile. For more description, Fig. 5 show how its works.

\subsection{Acoustic Advantage}

The advantage using this technique is that there is no other in-water acoustic equipment has to deploy before underwater operations can commence. Rather than ordinary technique that need the targets that being tracked need to be equipped with a transponder

\subsection{Acoustic Disadvantage}

The most common problem for acoustic navigation comes from the position of thruster location on vehicle that produces noise. The thruster noise, it effect the optimum measurement of the acoustic signal, this called the echo range, where the error occur on timing, the error occur on calibration method. The range and offset angel also one of the factor of the error. The Fig. 6 below show the position error when distance from transducer to thruster is $15 \mathrm{~m}$ and $25 \mathrm{~m}$ for LBL array.

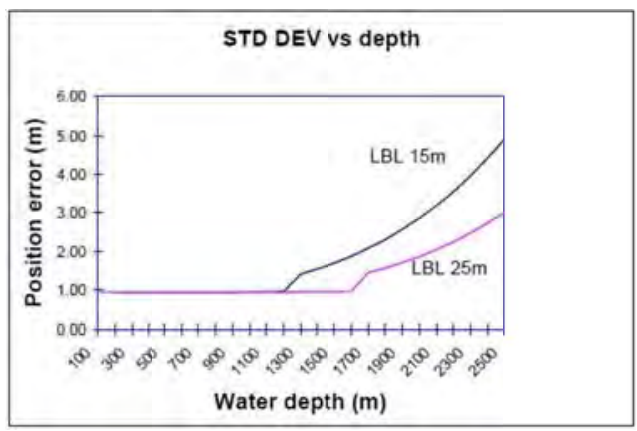

Fig. 6. LBL Graph of accuracy vs depth

\section{Geophysical Navigation}

Geophysical Navigation usually focused on environmental map and its matching with priori sensor data and the assumption which is considered in this matching is to get accurate localization by measuring sufficient spatial variation in the parameter. So in actual, the geophysical environment is measurement of geophysical parameters, such as bathymetry, magnetic field, or gravitational anomaly $[17,18]$.

As shown in Fig.4 the black bold line is the vehicle path, and the outline of Bottom Ocean is mapping base on its altitude.

The process is very simple: First, the real time information is collect to match the environment on database. When the UUV match with database then it knows its location on map.

In reality this method is not a straightforward technique because the UUV is attempting to navigate by matching a set of sensed data with a priori map, the main problem is the generation of the priori maps and the computational complexity of searching a peak of correlation surface.

The geophysical navigation algorithm has a technique of navigation at ocean using depth sounding that has been in use for centuries. The maps from the real time sensor is matching with the data from the database, the contour line that intercept with both data is consider the 
correct matching of computer vision latter it will determine the extract location of UUV. The accuracy of this technique is depending on the priori map. The mapping technique is show in the Fig. 7.

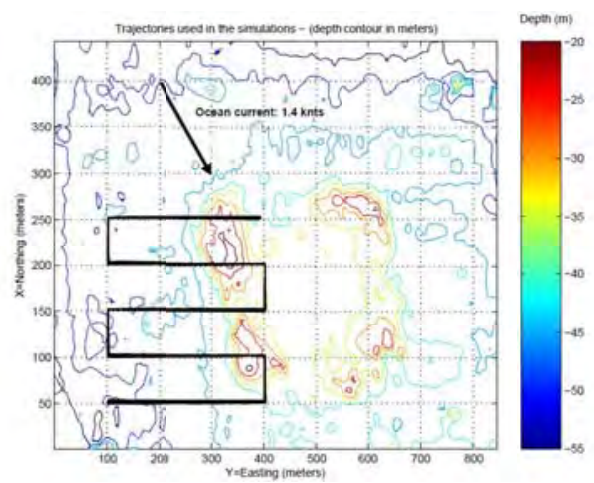

Fig.7. The mapping of terrain using geophysical navigation

\subsection{Geophysical Navigation Advantage}

The main benefit of using the geophysical navigation is it's have the large operating range where can be use. The technique is completely onboard navigation capability unrestricted by range to external sensor [13]. Furthermore, the navigation is only restricted by the range of its sensor used for geophysical measurement, the operating range of this technique is also very large. the navigation also can work on highly unconstructed environment.

\subsection{Geophysical Navigation Disadvantage}

The geophysical navigation suffers from several error that come from the mapping technique itself, the measurement are defined to the terrain map. The approach of the vehicle need to be in a right situation to allow the map is mapping with a high precision condition. One of the errors is come from the difference between the map and inertial reference frame which arises in part from imperfect navigation during map generation.

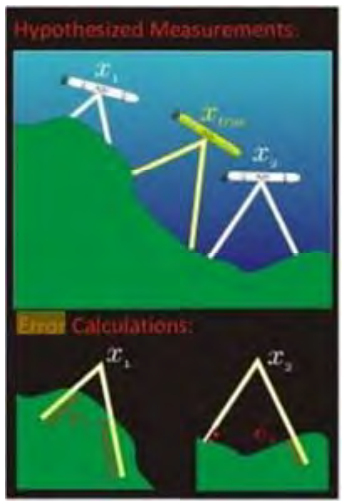

Fig. 8. Range Base Error

\section{Global Positioning System (GPS) Navigation}

For navigation, the GPS is widely use in various application. This navigation is a type of satellites information that provides world-wide position information. It does commonly give accuracy near to \pm 100 meter at $95 \%$ confidence level. Unfortunately in underwater vehicle, this system cannot continuously give an information because this signal cannot be capture by receiver because the there is no water penetrating capability. To GPS fix its location, the antenna must be out of water, this by extending the antenna or by moving the UUV to surface. The operational aspect of this system is also not a popular choice for UUV because the surfacing operation in UUV will scarified the time and power consumption, the mission will also been interrupted, sometimes the operation when surfacing need to consider the positioning error. It's also not very suitable to surfacing at the busy area such as harbor that highly possibility can cause a collision between the surface ships.

\subsection{GPS Navigation Advantage}

The Global Positioning System (GPS) is the most widely use application in many of autonomous vehicle, the main advantage of this technique; the accuracy is a pin point location for high grade GPS, the position is near to actual value. This technique is also use to correction of other navigation system, where the positioning of GPS will be the main reference to 
D. Hazry, S. Faiz Ahmed, M. Hassan Tanveer, M. Kamran Joyo, Faizan. A. Warsi,

Khairunizam Wan, Zuradzman M. Razlan, Shahriman A.B, Zunaidi IB and A.T. Hussain

determine the extract location, the reliability of this technique is very robust.

\subsection{GPS Navigation Error}

The typical problem of GPS navigation system is the signal have no penetrating under water, so when the vehicle is submerged, the signal is lost, and the vehicle is need a other technique to keep in mission, other subject of this error is the GPS have their loading signal where when resurfacing, usually its take times to detect the signal, and sometime the signal is lost. The GPS also have an error from the satellite position, which are delusions of precision (DOP) where when this happen the position is absolutely incorrect, the situation is show in the Fig. 9.

\section{The Comparison of Navigation Methods}

In this section all four discussed navigation techniques are compared with each other in different mission range situations. For near costal or short ranged mission, inertial navigation system gives better results, regardless of the path taken by the UUV, but when greater accuracy is required, the acoustic navigation system are more suitable. For long range navigation, acoustic navigation method can be used with SLAM (Simultaneous localization \& mapping) technique to correct the inaccuracies in the UUV position. It compares the UUV current position with Mapped crossover points during maneuvering while crossing the mapped points. In this method number of crossover points determines the efficiency of navigation system and limited up to sequential registration landmarks.

For the very long range mission, deployment of large network of crossover point with mapping of their location is difficult and the vehicle experiences the significant drift when used this method. Geophysical navigation system can be used in very long range missions, however, the limited source of available maps make this technique very difficult and not reliable for very large area. GPS navigation gives better results over this range to determine the current position, but the vehicle need to constantly resurface to correct its position, which this limitation compare to other method GPS navigation system can work in all the mission more accurately and efficiently. Table 1 is show the comparison chart for all four navigation methods in mission range conditions.
Table 1. The Accuracy Table base on mission range.

\begin{tabular}{|l|lll|}
\hline $\begin{array}{l}\text { Navigation } \\
\text { Technique }\end{array}$ & Mission Range & \\
& SHORT & LARGE & VERY \\
& & & LARGE \\
\hline Dear Reckoning & $\bullet$ & $\bullet$ & \\
\hline Acoustic & $\bullet$ & $\bullet$ & \\
\hline Geophysical & & $\bullet$ & \\
\hline GPS & & $\bullet$ & \\
\hline
\end{tabular}

\section{Conclusion}

In this article different technique for underwater navigation has been presented along with concise comparison of their suitability for the difference mission tasks. The current treads of UUV navigations have been recognized and throughly discussed their advantages and disadavatages in different mission tasks. Finally the comparison has been done for different navigation techniques in range mission situations and found that the inertial navigation method, global positioning system and acoustic routines navigation techniques are more developed and better as compare to geophysical routine navigation method. We can conclude that for the Aqua-X UUV application, GPS navigation method is best suitable technique because it can work in all types of mission tasks i.e. Short, Large as well as very large missions.

\section{References}

1. Atkins, W. A. (2011). Submarines and Submersibles.Water Encyclopedia, from http://www.waterencyclopedia.com/St-

Ts/Submarines-and-Submersibles.html. Retrieved 2 August 2010.

2. Grant, M. S. (2010). Reef Explorer Hovering Autonomous Underwater Vehicle: AUV Laboratory [Electronic Version].

3. Johnson, D, Eppig, S, "Aided intertial navigation system for underwater vehicles". Proceedings of the 5thInt, Symposium on Unmanned Untethered 
Submersible Technology, New Hampshire, USA, 1987.

4. Geyer, E.M. et al, "Characteristics and capabilities of navigation system for unmanned untethered submersibles", Proceedings of the 5th int, Symposium on Unmanned Untethered Submersible Technology, New Hampshire, USA, 1987

5. Bellingham, J.G et. Al, "Perfomance Characteristic of the Odyssey AUV", Proceedings of the 8th Int, Symposium on Unmanned Untethered Submersible Technology, NewHampshire, USA, 1993

6. Bellingham, J.G ET. AL, Artic Uderwater-Ice survey Operations", Proceedings of the 8th Int, Symposium on Unmanned Untethered Submersible Technology, New Hampshire, USA, 1993

7. Butler, B, Hertog, V, "Theseus: a Cable-Laying AUV”. Proceedings of the 8th Int, Symposium on Unmanned Untethered Submersible Technology, New Hampshire, USA, 1993

8. Mindell, D.A and Yoerger D. “ A Hybrid Parallel Control and Navigation System for the Autonomous Bentic Explorer (ABE) vehicle", Peoceedings of the 8th int. Symposium on Unmanned Untethered Submersible Technology, New Hampshire, USA, 1993.

9. Elbert S. Maloney, editor. Dutton's Navigation and Piloting. Annapolis, MD: Naval Institude Press, 1985

10. M. Kuristsky and M. Goldstein. Inertial navigation. In I. Cox and G. Wilfong, editors, Autonomous Robot Vehicle. Springer-Verlag, 1990.

11. J. G .Paglia, W. F . Wayman. DARPA's Autonomous Minehunting and Mapping Technologies(AMMT) Program: An overview. In IEEE Oceans, pages 749-799,Ft Launderdale, FL, USA, September 1996.

12. J . G Bellingham, T .R Consi, U. Tedrow, and D. Di Massa. Hyperbolic acoustic navigation for underwater vehicle: Implementation and demonstration, In Proceedings AUV. 92, pages 304309, 1992.

13. Meduna, D. K. (2011). Terrain relative navigation for sensor-limited systems with application to underwater vehicles. Stanford University.

14. Jalving, B., Gade, K., Hagen, O. K., \& Vestgard, K. (2003, September). A toolbox of aiding techniques for the HUGIN AUV integrated inertial navigation system. In OCEANS 2003. Proceedings (Vol. 2, pp. 1146-1153). IEEE.

15. Hofmann-Wellenhof, B., Lichtenegger, H., \& Wasle, E. (2008). Gnss: Global Navigation Satellite Systems: Gps, Glonass, Galileo, and More. Springer.

16. Tanveer, M. H., Ahmed, S. F., Hazry, D., Warsi, F. A., \& Joyo, M. K. (2013). STABILIZED CONTROLLER DESIGN FOR ATTITUDE AND ALTITUDE CONTROLLING OF QUAD-ROTOR UNDER DISTURBANCE AND NOISY CONDITIONS. American Journal of Applied Sciences, 10(8), 819 